Article

\title{
The Strengthening Effect of Phase Boundaries in a Severely Plastically Deformed Ti-Al Composite Wire
}

\section{Tom Marr ${ }^{1,2}$, Jens Freudenberger ${ }^{1,3, *}$, Verena Maier ${ }^{4, \dagger}$, Heinz Werner Höppel ${ }^{4}$, Mathias Göken ${ }^{4}$ and Ludwig Schultz ${ }^{1,2}$}

${ }^{1}$ IFW Dresden, Institut für Metallische Werkstoffe, Helmholtzstraße 20, Dresden 01069, Germany; E-Mails: t.marr@ifw-dresden.de (T.M.); 1.schultz@ifw-dresden.de (L.S.)

${ }^{2}$ TU Dresden, Institut für Werkstoffwissenschaft, Dresden 01062, Germany

${ }^{3}$ TU Bergakademie Freiberg, Institut für Werkstoffwissenschaft, Gustav Zeuner Straße 5, Freiberg 09599, Germany

${ }^{4}$ Department Werkstoffwissenschaften, Lehrstuhl für Allgemeine Werkstoffeigenschaften, Universität Erlangen-Nürnberg, Martensstr. 5, Erlangen 91058, Germany;

E-Mails: verena.maier@unileoben.ac.at (V.M.); hoeppel@ww.uni-erlangen.de (W.H.); goeken@ww.uni-erlangen.de (M.G.)

${ }^{\dagger}$ Current Address: Montanuniversität Leoben, Institut für Materialphysik, Jahnstr.12, A-8700 Leoben.

* Author to whom correspondence should be addressed; E-Mail: j.freudenberger@ifw-dresden.de; Tel.: +49-351-4659550.

Received: 26 November 2013; in revised form: 16 January 2014 / Accepted: 23 January 2014 / Published: 7 February 2014

\begin{abstract}
An accumulative swaging and bundling technique is used to prepare composite wires made of $\mathrm{Ti}$ and an $\mathrm{Al}$ alloy. These wires show reasonable higher yield stresses than expected from the pure material flow curves. The additional strengthening in the composite is analyzed using nanoindentation measurements, tensile testings and investigations of the microstructure. In addition, these properties are analyzed in relation to the fracture surface of the mechanically tested wires. Additional strengthening due to the presence of phase boundaries could be verified. Indications for residual stresses are found that cause a global hardness gradient from the center to the wire rim. Finally, the yield stress of the wires are calculated based on local hardness measurements.
\end{abstract}

Keywords: SPD; Ti; Al; nanoindentation; mechanical properties 


\section{Introduction}

In the past few decades, a number of different methods has been established in order to deform materials to a very large plastic strain. Consequently, the methods involved, as well as the materials being processed are related to and described in terms of severe plastic deformation (SPD). The mentioned methods cover commonly used methods, such as equal channel angular pressing (ECAP) [1-3], high pressure torsion (HPT) [4,5] or accumulative roll bonding (ARB) [6-9], as well as comparably less applied ones, such as, e.g., multi-directional forging [10,11] or twist extrusion [12].

These methods allow the preparation of bulk ultra-fine grained (UFG) or even nano-crystalline materials [13]. However, co-deforming composites in a well defined way is a difficult task for most of these methods. Therefore, most of the materials that have been processed by SPD-methods are single component materials. The ARB process is an exception that allows homogeneous co-deformation of different phases of one material or even of different materials up to a certain extent [14-17].

Another exception to applying SPD is represented by an accumulative swaging and bundling process (ASB). This process has recently been applied on Ti-Al composites [18,19]. The present investigations were also performed on composites made from Ti and an Al alloy being co-deformed by ASB up to strains that reveal a UFG microstructure. These wires show extraordinary properties, since their yield stress is found to be higher than expected from a combination of the yield stress values of the individually deformed materials.

Therefore, the phase boundaries introduced by ASB are expected to be responsible for the additional strengthening. They are studied in detail using nanoindentation hardness measurements. It is found that phase boundaries play a crucial role when dealing with this kind of composite. In combination with tensile testings, microstructural analysis and fracture surface analysis, a comprehensive picture of the mechanical behavior of such composites is drawn. This is thought to be necessary, since the observation of an additional strengthening effect by phase boundaries opens up the opportunity to further enhance the mechanical properties of SPD materials.

\section{Experimental Section}

The material studied was processed by co-deforming an Al alloy 5049 rod within a Ti grade 1 tube accumulatively via rotary swaging. The preparation route has been reported earlier [18,19]. Without any re-stacking, such a composite is denoted as TiAl0, where the number corresponds to the number of already applied stacking cycles. After one, two or three applied stacking cycles, samples are referred to as TiAl1, TiAl2 and TiAl3, respectively. Due to stacking pre-deformed into un-deformed material and subsequently co-deforming both, the cross-section of the final composites consists of differently pre-strained areas, as shown in Figure 2. Tensile tests were carried out at room temperature using an Instron 8502 tensile testing machine in strain controlled mode at a constant initial strain rate of $3.3 \times 10^{-4} \mathrm{~s}^{-1}$.

Flow curves were obtained by deforming the single materials by rotary swaging at room temperature in passes of $20 \%$ area reduction per pass up to a log deformation strain of 4.5.

The nanoindentation experiments were performed on a Nanoindenter G200 (Agilent Technologies, Chandler, AZ, USA), using a three-sided Berkovich diamond and a continuous stiffness measurement 
(CSM) technique. Tip shape calibration was performed according to the Oliver-Pharr method [20,21], and the machine compliance was taken into account. Using a standard CSM method, the indentation stiffness is determined continuously, and the reduced modulus and indentation hardness can be obtained as a function of the indentation depth.

Nanoindentations were performed as constant strain-rate controlled $\left(0.05 \mathrm{~s}^{-1} \mathrm{~s}\right)$ to different preset indentation depths $(100,500$, as well as $1000 \mathrm{~nm})$. Arrays of $3 \times 30$ indents were directly positioned over the $\mathrm{Al} / \mathrm{Ti}$ interfaces [22]; thereby, neighboring indentations were separated by a minimum distance of 20 times the maximum indentation depth to avoid the influence of the resultant plastic zone around every indentation [23]. The depth-dependent hardness was averaged between 80-90 nm or $400-450 \mathrm{~nm}$, respectively.

Scanning electron microscopy (SEM) was conducted using a Zeiss Gemini 3500 LEO microscope set up with a field emission gun operating at $20 \mathrm{kV}$ acceleration voltage in SE and BSE modes.

The distances of single indentations to phase boundaries were examined using SEM images of the indentation arrays using standard image analysis software.

It should be kept in mind that many of the discussed measures in the present study, such as stresses or strains, are tensors. Nevertheless, for a possible application, the most important measures are the properties in wire direction, such as yield stress or Young's modulus. Discussing them as scalars with respect to the wire axis (as it is often done in this study) sounds therefore reasonable, but when interpreting the data, it should be kept in mind that the underlying problem is of a higher dimensionality.

\section{Results and Discussion}

\subsection{Flow Curves of the Individual Materials and the Composite}

Figure 1 shows the flow curve of Ti grade 1, AA 5049 and the composite made from both materials. The flow curves of the individual materials are approximated using Ludwik's law [24]:

$$
\tau_{L}(\rho)=\tau_{0}+k \rho^{n_{L}}
$$

Herein, $\tau_{L}(\rho)$ and $n_{L}$ are the true yield stress and hardening exponents after Ludwik, respectively, $\tau_{0}$ and $k$ represent material-dependent constants and $\rho$ the true strain. Furthermore, the expression presented by Hockett and Sherby [25] is alternatively used for fitting the experimental data:

$$
\tau_{H}(\rho)=\tau_{s}-\exp \left(-(N \rho)^{p}\right)\left(\tau_{s}-\tau_{y}\right)
$$

Herein, $\tau_{H}(\rho)$ represents the true yield stress after Hockett, $\tau_{s}$ the saturation flow stress for infinite strain, $N$ and $p$ are free parameters and $\tau_{y}$ represents the yield stress at zero strain.

The commonly used power-law given by Hollomon [26] also fits the presented data, primarily resulting in lower hardening exponents and significantly lower correlation than the two models shown before. Hence, only Ludwik's law and Hockett's equation are discussed in this study. 
Figure 1. Flow curves of the used Ti grade 1, AA5049 alloy up to a true strain of 4.5 with fits by Ludwik's equation, the composite made from both materials up to a true strain of 16.6 and the calculated flow curves of the composites (preserved by weighting the fitted yield stresses for $\mathrm{Ti}$ and the $\mathrm{Al}$ alloy according to their volume fraction in the composite).

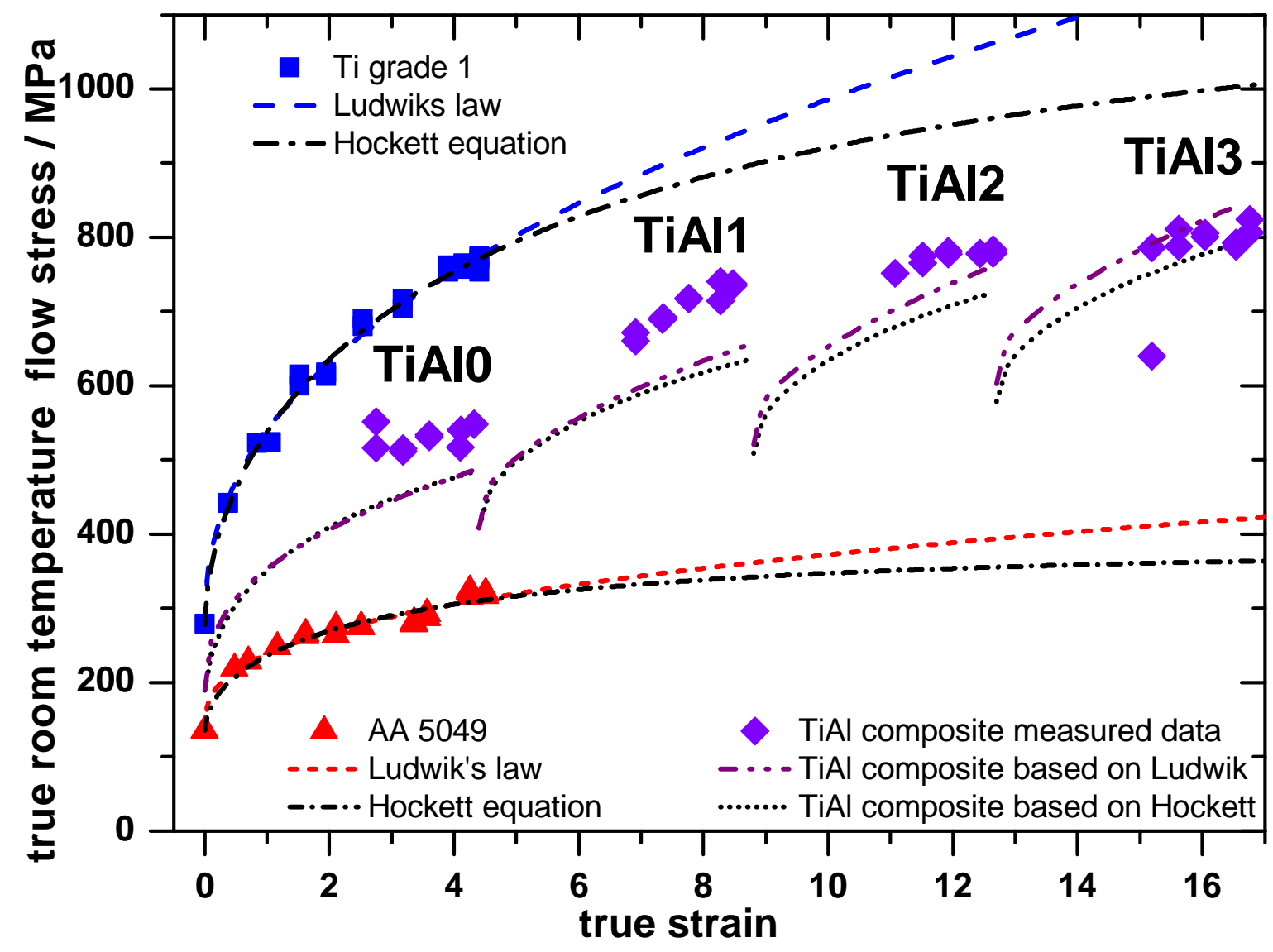

The derived parameters for Ti and AA5049 are given in Table 1 according to both equations. In the case of the $\mathrm{Al}$ alloy, Hockett's equation converges in a parameter set that exactly reproduces Ludwik's law, taking an unrealistically high saturation stress into account. Therefore, the exponent, $p$, was fixed for the calculation to 0.58 . This is believed to be reasonable, since Hockett found exactly the same value experimentally for $\alpha$-uranium and Armco iron. In this study, the same value was found to give very good agreement with experimental data for the used commercially pure titanium, even though all three materials differ entirely in their structure.

Obviously, both power-laws highly correlate with the measured flow curve data in the region $0 \leq \rho \leq 5$. Hence, both are extrapolated to a true strain value of 17 in order to estimate the hardening behavior of the individual materials up to very high deformation strains (like is reached for the composite using accumulative swaging and bundling). This is done since the individual materials could not be deformed to such high values without introducing significant scatter, due to the resulting small cross-section.

As the composite material is prepared by accumulative swaging and bundling, the cross-section of each composite differs in composition, because of the new Ti shell that is added to the composite within each stacking cycle. Nevertheless, during each deformation route up to the point where a new stack is formed, the volume fraction of the different phases and the differently strained parts of the cross-section 
are constant. The corresponding volume fraction is estimated by combining the results of mass density measurements and area analysis by optical microscopy and are given in Table 2. The different parts of the cross-section are exemplarily shown for clarity in Figure 2 for the TiAl0 and TiAl 2 composite. In the latter one, just before the new stacking, the $\mathrm{Al}$ alloy has experienced a true strain of 12.6, while the Ti shell, which was not introduced before the last stacking cycle, is only strained to a true strain of 4.3 in that composite. The different straining of the material results in different hardening and, therefore, locally different flow stresses, which are believed to follow the extrapolations shown in Figure 1.

Table 1. Fitting parameters used for fitting flow curves according to Equations (1) and (2).

\begin{tabular}{cccc}
\hline & Parameter & Ti & AA 5049 \\
\hline & $\tau_{0} / \mathrm{MPa}$ & $275.5 \pm 12.4$ & $137.4 \pm 9.1$ \\
Ludwik's law & $k / \mathrm{MPa}$ & $263.8 \pm 13.4$ & $101.0 \pm 9.9$ \\
& $n_{L}$ & $0.4301 \pm 0.022$ & $0.3665 \pm 0.040$ \\
& $\mathrm{R}^{2}$ & 0.99 & 0.95 \\
\hline Hockett's eq. & $\sigma_{s} / \mathrm{MPa}$ & $1139 \pm 282$ & $380 \pm 28$ \\
& $\mathrm{~N}$ & $0.17 \pm 0.15$ & $0.33 \pm 0,11$ \\
& $\sigma_{y} / \mathrm{MPa}$ & $0.583 \pm 0.083$ & $0.58^{*}$ \\
& $\mathrm{R}^{2}$ & 0.99 & $135.5^{*}$ \\
\hline
\end{tabular}

* fixed, see text.

Table 2. Volume fractions of the different deformed parts of the composites, TiAl0, TiAl1, TiAl2 and TiAl3. Data is derived by mass density measurements and quantitative analysis of the phase arrangements. For errors, please refer to the error calculation in the supplementary.

Ti

AA 5049

\begin{tabular}{ccccccccc}
\hline true strain & $\mathbf{0 - 4 . 3}$ & $\mathbf{4 . 3 - 8 . 5}$ & $\mathbf{8 . 5 - 1 2 . 6}$ & $\mathbf{1 2 . 6 - 1 6 . 8}$ & $\mathbf{0 - 4 . 3}$ & $\mathbf{4 . 3 - 8 . 5}$ & $\mathbf{8 . 5 - 1 2 . 6}$ & $\mathbf{1 2 . 6 - 1 6 . 8}$ \\
\hline \multirow{2}{*}{ TiA10 } & 0.381 & 0 & 0 & 0 & 0.619 & 0 & 0 & 0 \\
& & & & & \pm 0.022 & & & \\
\multirow{2}{*}{ TiAl1 } & 0.374 & 0.239 & 0 & 0 & 0 & 0.387 & 0 & 0 \\
& \pm 0.009 & \pm 0.012 & & & & \pm 0.008 & & \\
\hline \multirow{2}{*}{ TiAl2 } & 0.373 & 0.239 & 0.159 & 0 & 0 & 0 & 0.23 & 0 \\
& \pm 0.007 & \pm 0.012 & \pm 0.014 & & & & \pm 0.003 & \\
\hline \multirow{2}{*}{ TiAl3 } & 0.359 & 0.239 & 0.159 & 0.108 & 0 & 0 & 0 & 0.137 \\
& \pm 0.007 & \pm 0.012 & \pm 0.014 & \pm 0.02 & & & & \pm 0.002 \\
\hline
\end{tabular}


Figure 2. Visualization of the differently strained parts of the cross-section of the two composites, TiAl0 and TiAl2 (without stacking and two times stacked, respectively), in back scattered electron images. The given numbers correspond to the true strain of the cross-section shown.
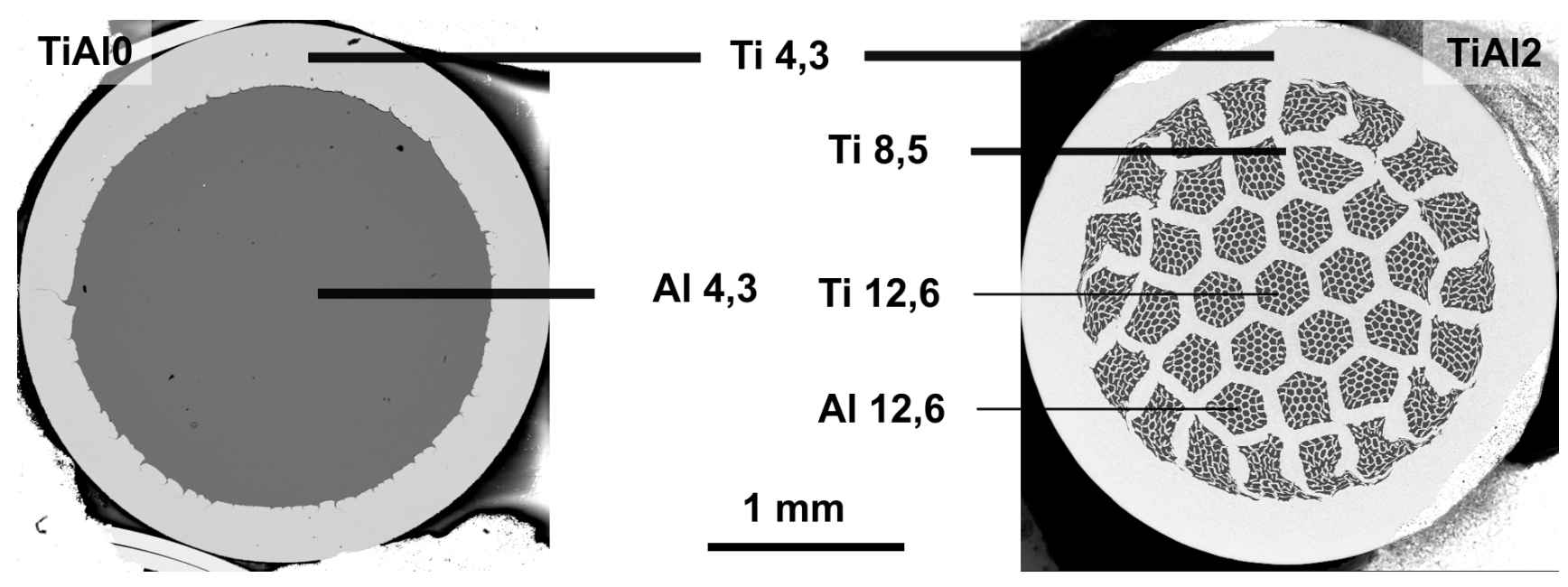

In a simple attempt to calculate the yield stress of the composite based on the yielding behavior of the individual materials, the yield stress of each individual part of the wires is weighted by the corresponding volume fraction (Table 2) and, thus, adds to the calculated yield stress of the entire wire. Data obtained in such a way is also illustrated in Figure 1 (violet: Ludwik; black: Hockett).

The value of the yield stress being calculated for the composite upon Hockett's equation (Equation (2)) fits the experimental data for very high strain (14-17) very well, while Ludwik's law seems to overestimate the yield stress in that region. This sounds plausible, since Hockett's equation was originally introduced to describe a material flow stress at very high deformation strain levels, which is also the case here.

Furthermore, the composites, TiAl0 (unstacked) and TiAl1 (stacked once), show yield stresses well above the calculated ones, even in the range of $0 \leq \rho \leq 5$, where measured, but not extrapolated, data of the individual materials is presented. This is not expected, since phase boundaries, as they are introduced in the composite, usually weaken the composite, due to inhomogeneities, contaminations, oxide layers or small cracks having a negative influence on the entire compound all together. In Figure 2, such cracks are even visible over the full length of the phase boundary. Even though these negative influences should decrease the composites' mechanical properties, due to the reduction of the effective cross-sectional area, the measured yielding point is (in places) up to $100 \mathrm{MPa}$ higher compared to the calculations. Hence, the introduced phase boundary also strengthens the composite in some way.

This strengthening effect seems to decrease with increasing accumulated strain or with more phase boundaries being introduced, as differences between the calculated and the measured data is reduced for the composites, TiAl2 and TiAl3. Hence, the additional strengthening does not primarily scale with the number of phase boundaries. Apparently, their mean distances have to be taken into account as characteristic length scales.

These mean distances between phase boundaries for the composites are: TiAl0: $2.2 \mathrm{~mm}$; TiAl1: $280 \mu \mathrm{m}$; TiAl2: $35 \mu \mathrm{m}$; and TiAl3: $5 \mu \mathrm{m}$. The strengthening effect is strongest for the first two, 
where characteristic length scales are well above $100 \mu \mathrm{m}$. This indicates that the operating strengthening mechanism does not act as simple dislocation barriers, which would be comparable with grain boundaries in terms of the Hall-Petch relationship. The observed strengthening is more effective at larger length scales.

\subsection{Nanoindentation of Each Wire Part}

To study the observed strengthening effect in more detail, nanoindentation measurements were deployed at the different parts of each composite. This attempt was made to find out whether a local hardness increase near phase boundaries could be a possible origin of the effect.

By using this technique, it is possible to study local hardness effects near phase boundaries and according to the overall position in the wire (e.g., the distance to the wire axis). The Ti part of the composites increases in hardness depending on the absolute true strain level (Figure 3a). It is observed that $\mathrm{Ti}$ with the same nominal true strain level shows a lower hardness near the wire rim than at the center. Almost the same behavior is found for the $\mathrm{Al}$ alloy.

Figure 3. Hardness of (a) the Ti and AA5049 parts of the wires strained to different strain levels. Open symbols show hardness near the wire rim, while filled symbols correspond to the wire center region. (b) The hardness of Ti bridges deformed to a true strain of 8.5; no phase boundary effect within $\mathrm{Ti}$ is visible. Filled and open symbols are shifted manually to different values for clarity reasons. Each of them correspond to the true strain values of 4.3, $8.5,12.6$ and 16.8 , respectively.

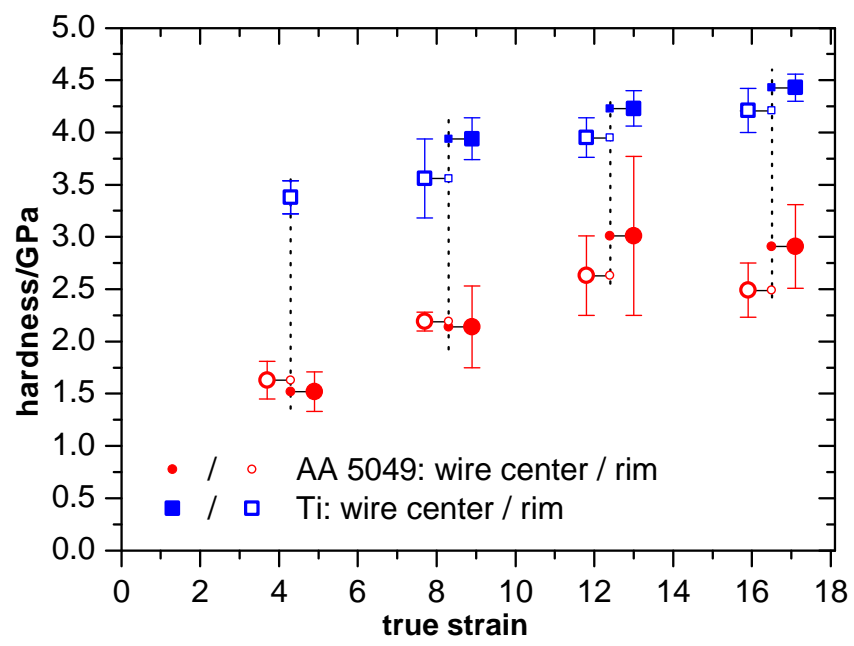

(a)

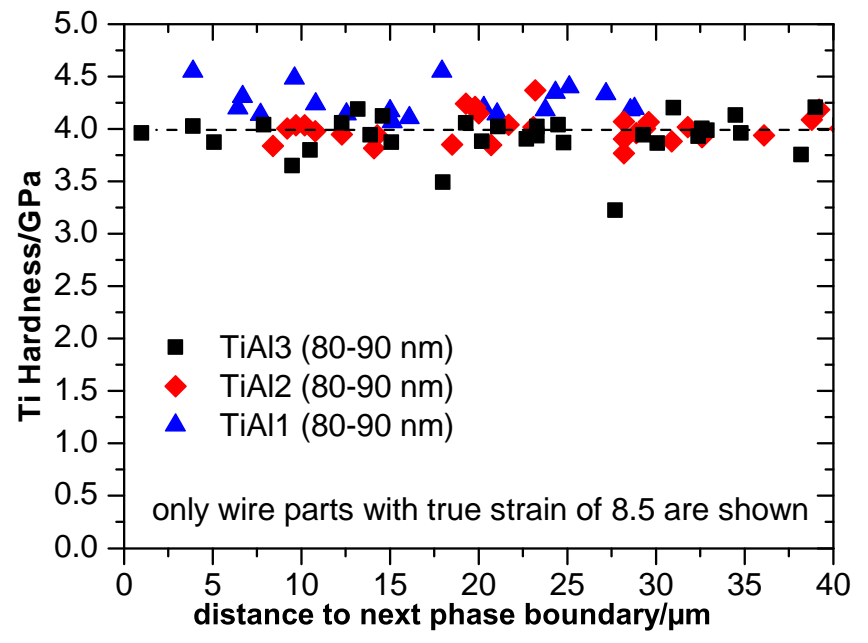

(b)

The wire center is the region where no additional shearing during rotary swaging occurs; therefore, deformation is homogeneous up to the very end of the deformation route. This is not the case for regions near the wire rim, where additional shear deformation takes place, due to friction with the rotating forming tools. This is believed to be one of the reasons for the lower hardness in this region, but temperature might also play a role. Taking Titanium's low thermal conductivity into account, it seems plausible that heat can accumulate during deformation within the Ti-rich regions near the wire 
rim. This can lead to a more pronounced tendency for recovery mechanisms. Nevertheless, further studies with sacrificed thermocouples on temperature development during deformation demonstrate that the maximum temperature reached during deformation is approximately $300{ }^{\circ} \mathrm{C}$ for just a few seconds [27]. Significant recovery in Ti is therefore unlikely to cause the lower hardness near the wires' rim. Another possible explanation for this observation is a texture gradient or a certain residual stress state that differs from the center to the rim of the wires.

Within one certain $\mathrm{Ti}$ part, there is no change in hardness visible, when a phase boundary is approached. This is shown in Figure $3 \mathrm{~b}$ for the Ti bridges strained to a true strain of 8.5 in the different composites that have these bridges available. The mean hardness value is about $4 \mathrm{GPa}$ and is roughly the same for all composites. Only hardness in TiAl1 is slightly increased compared to the others.

In other words, the hardness in Ti and AA5049 correlates with the true strain, but there is no phase boundary-driven hardness increase or decrease visible in the Ti parts of the composites. The wire center is, in all cases, slightly harder than comparable regions near the rim. Since the latter observation is found for all composites, it can be excluded to be the only reason for the higher-than-expected composite yield stresses in Figure 1, since the difference to the expected values is strain dependent.

At very low strains, Müller found the radial strain in single component materials deformed by the present process to be at a small maximum near the wire axis [28]. A higher stain compared to the rim could explain the higher hardness near the wire axis. Nevertheless, at high strains, as they are obtained here, this effect should be neglected, due to the marginal slope of the flow curve at high strains. It is therefore assumed that the hardness gradient in $\mathrm{Ti}$ and $\mathrm{Al}$ from the center to the rim is the result of a complex residual stress state.

At this point, it should be mentioned that hardness measurements will only be sensitive for radial and circumferential stresses, since axial residual stresses disappear when cutting the wires for preparation. However, they give rise to the assumption of a complex residual stress state, which will have an influence on hardness measurements, as well as the global yield stress. A direct measurement of these stresses is difficult. By neutron measurements, it is not possible to distinguish between the differently strained parts, and the composite itself causes problems, as well. Local strain measurements by X-ray measurements suffer from the texture in $\mathrm{Ti}$ (the $c$-axis is aligned parallel to the radial direction) and the bad FWHM (full width half maximum) of the reflexes, due to the high strain. Finite element modeling might help in this case, but the problem is still quite complex, due to the wires' structure.

In Figure $4 a, b$, hardness values of the different strained parts of the Al alloy are shown. In the TiAl3 composite, phase boundary distances approach the residual impression size for higher indentation depths. At the same time, comparable data for all composites is needed. Hence, all measurements were averaged in a range between 80 and $90 \mathrm{~nm}$ of indentation depth. Of course, this introduces more scatter, especially for the lower strained composites, but all measured hardness data is directly comparable. To give an impression of the depth-dependent hardness [29], Figure 4a also shows that the hardness data of TiAl0 averaged between $400-450 \mathrm{~nm}$. With increasing indentation depth, the hardness slightly decreases, due to a less pronounced indentation size effect (ISE). Since the investigated grain structure is ultra-fine grained, a reduced ISE and, thus, a more or less depth independent hardness was expected, due to the reduced internal length scale in UFG- and nanocrystalline (NC) -materials [30,31]. 
Figure 4. Nanoindentation hardness of the AA 5049 alloy taken (a) near the wire shell and (b) near the wire center of all composites. Note that data points for 2TiAl0 are the same in (a) and (b), since there is only one phase boundary. Numbers in brackets indicate the indentation depth from which the hardness value is derived.

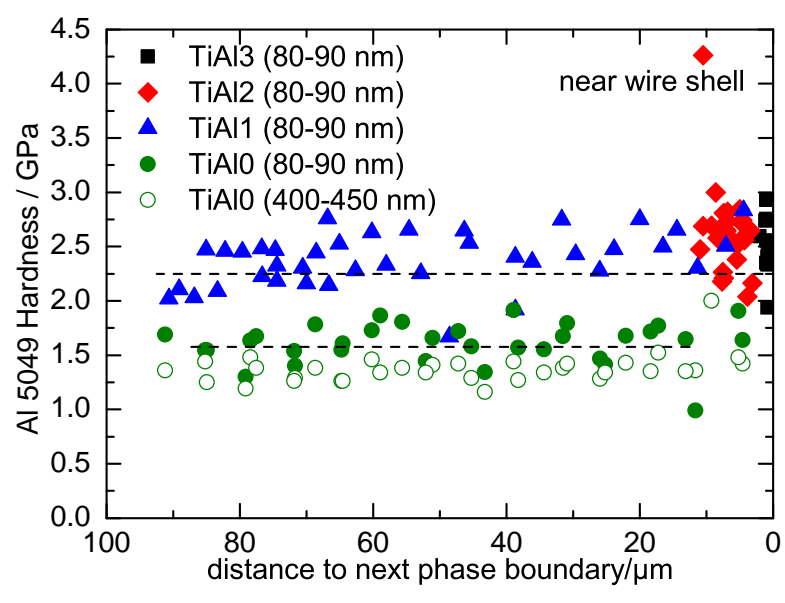

(a)

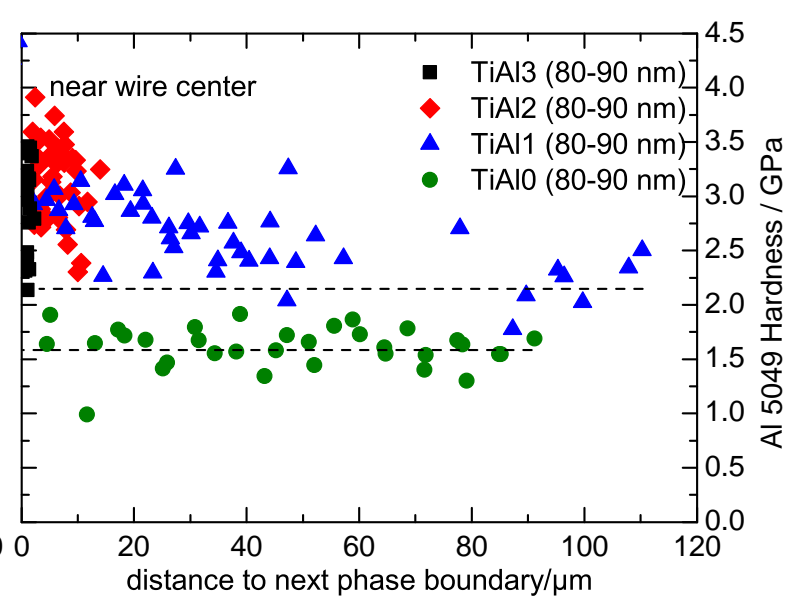

(b)

In the case of the TiAl0 composite, a slight increase in hardness with decreasing boundary distance is observed (from $c a$. $1.5 \mathrm{GPa}$ at the maximum distance to $c a$. $1.75 \mathrm{GPa}$ near the boundary). This is a little less pronounced for the data taken at $400-450 \mathrm{~nm}$ of indentation depth (from $c a$. 1.4 GPa to $c a$. $1.5 \mathrm{GPa})$.

The TiAll composite develops a hardness value of about $2.14 \mathrm{GPa}$ far from the next boundary. However, hardness increases next to a boundary to roughly $2.75 \mathrm{GPa}$ at the wire rim and almost to $3 \mathrm{GPa}$ near the wire center. This corresponds to an increase in hardness of about $25 \%-35 \%$ starting at a distance of about $70 \mu \mathrm{m}$ from the boundary.

The Al part of TiAl2 and TiAl3 shows no significant phase boundary effect, mainly due to the large scatter (compare the mean values in Figure 3a). Their hardness value seems to saturate just below $3 \mathrm{GPa}$ in the wire center and at $2.75 \mathrm{GPa}$ near the rim. This is almost the same hardness level that is reached by TiAll near the phase boundary. Therefore, it is not clear whether this hardness value is related to phase boundaries or to the higher strain, since the boundaries are already very close together. The Al microstructure in both cases has already saturated in grain size, and no grain size gradient within the Al filaments is observed.

Nevertheless, an increase in $\mathrm{Al}$ hardness next to a phase boundary can be proven. It is most pronounced in the TiAll composite, which is also the one with the largest differences in yield stress compared to the calculations. This is a sign that the local hardness increase is directly connected to the macroscopic yield strength enhancement.

Thereby, an influence of the measurement itself on the hardness increase can be neglected, since the effect already starts $70 \mu \mathrm{m}$ away from a boundary, while the indentation size at the analysis depth is below $1 \mu \mathrm{m}$. 


\subsection{Al Microstructure Near Phase Boundaries}

Figure 5 shows the Al microstructure far from a phase boundary (left side) for TiAl0 and TiAl1 and close to a boundary (right side). Without performing a detailed grain size analysis, it is obvious that the grain size changes not only from $\rho=4.3$ (TiAl0) to $\rho=8.5$ (TiAl1), but also with the distance to a boundary.

Figure 5. Back scattered electron images of Al microstructure in TiAl0 (upper row) and TiAl1 (bottom row); left side: far from the boundary; right side: close to the phase boundary

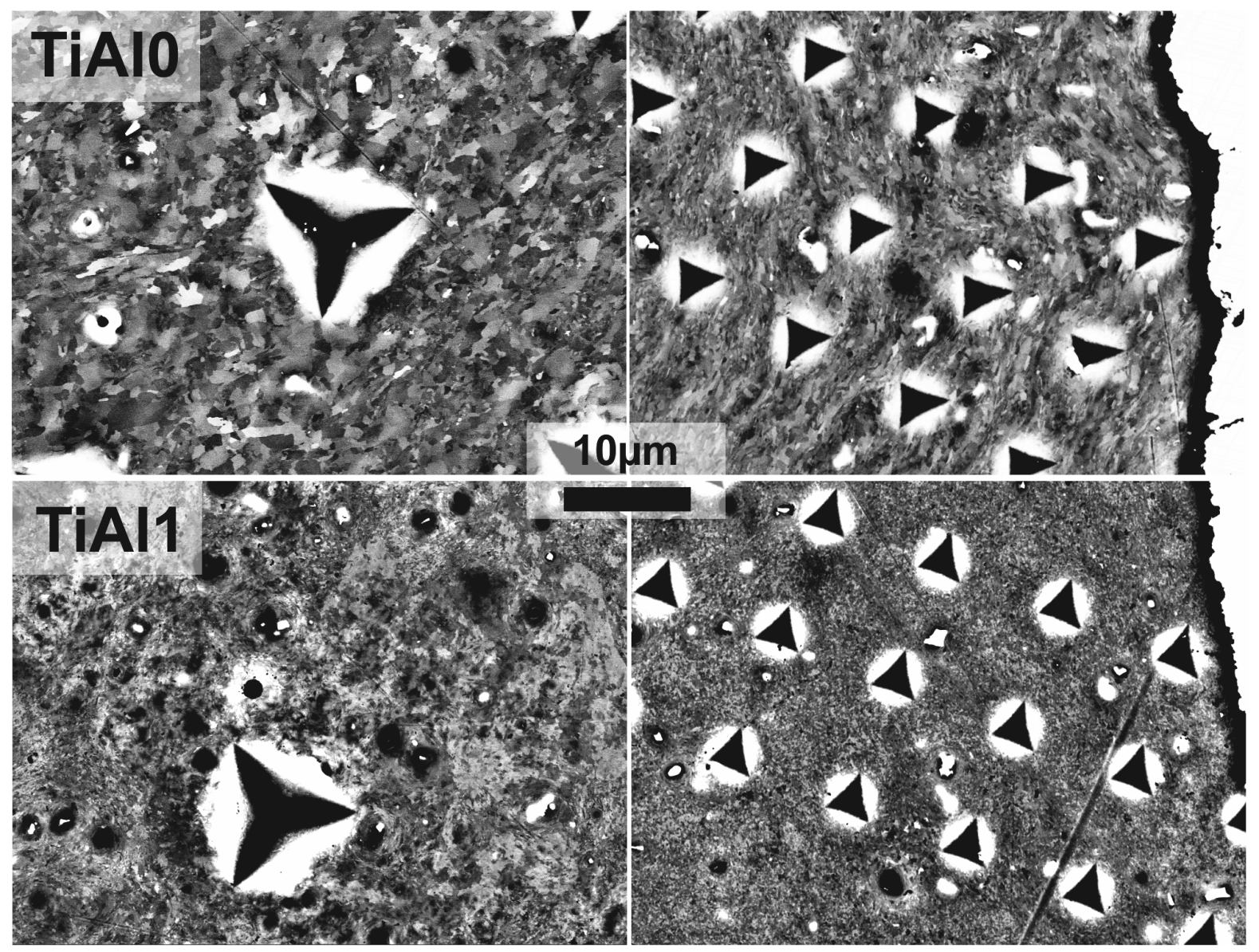

The grain size is a striking parameter in SPD processed materials. A decreasing grain size with strain is not surprising and is commonly put into relation with strength and hardness. Earlier studies reveal that the grain size is reduced from TiAl0 to TiAl1 from $0.8-3 \mu \mathrm{m}$ to $0.4-1.25 \mu \mathrm{m}$ (depending on the grain boundary definition), respectively [19]. This, however, only holds for regions far from boundaries. Here, it is evident that the grain size is further reduced next to a phase boundary. The additional shear deformation at the very outside of TiAl0 due to the influence of friction with the forming tools is a sound reason for this observation. Additional shearing causes a more complex displacement tensor in the affected region. This requires more active slip systems or, at least, the already active ones to be activated in a more distinctive way. Hence, a more pronounced dislocation-based hardening is the consequence. This is a possible explanation for the smaller grain size there, as well, since grain fragmentation during SPD is believed to be accompanied by dislocations, forming cell walls, followed by low angle grain boundary formation [32]. 
To sum up the previous statements, it is proposed, that the (local) hardness increase next to phase boundaries arises from: (i) higher dislocation activity due to localized additional shear deformation; and, in consequence, (ii) a smaller local grain size based on the advanced stage of grain refinement in these areas. However, the grain size might not be the most important parameter, since, for TiAl0, the grain size near the boundary is significantly reduced, but hardness is only slightly increased. The effect is more pronounced for TiAl1. Superimposed residual stresses are one explanation for the different behavior in TiAl0 and TiAl1 regarding the grain size; another would be a possible local change in texture. However, this is not addressed in the present study.

Since the filament size becomes very small for TiAl2 and TiAl3, a similar result is not found for these composites. For most filaments of these composites, neither a grain size nor a hardness gradient can be detected. Nevertheless, the decreasing hardness in the radial direction is still observed for $\mathrm{Ti}$ and $\mathrm{Al}$ in TiAl2 and TiAl3, which indicates that the underlying mechanism for the global hardness differences (from the wire center to rim) and the local (within a filament in TiAl1) mechanism is of a different nature.

\subsection{Fracture Surface Analysis}

An overview of the fracture surfaces for each composite after tensile testing is given in Figure 6. All wires show pronounced necking, which indicates the remaining ductility under the set testing conditions.

Figure 6. Top view of fracture surfaces after tensile testing of TiAl0-3.

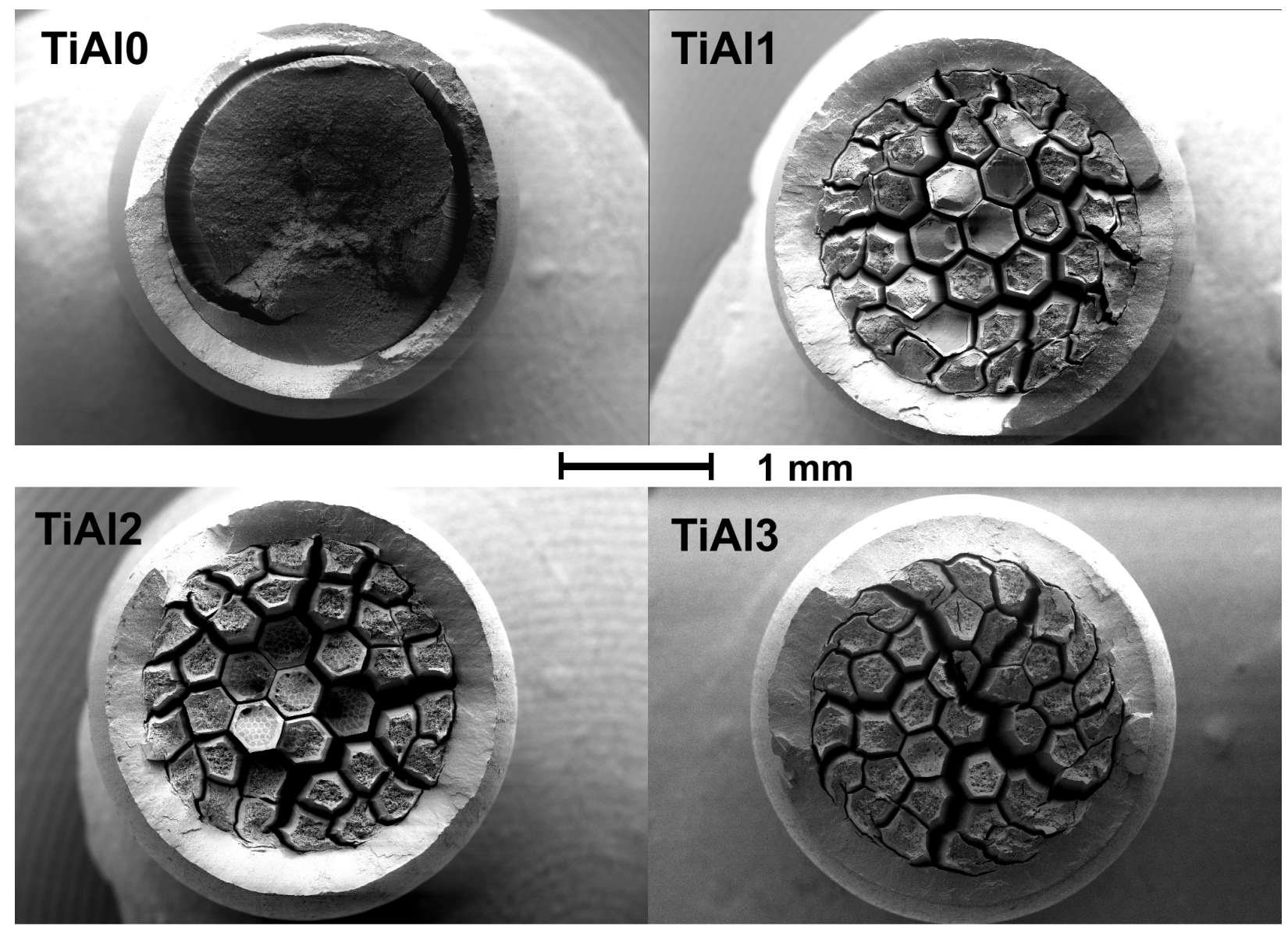


The $\mathrm{Al}$ core in TiAl0, as well as many of the filaments resulting from the last stacking cycle in the other composites even exhibit individual necking. This means, that almost all parts of the wires are loaded and yielding more or less simultaneously.

Interestingly, in the TiAl1 and TiAl2 composite, some of the filaments near the center show smooth surfaces and no or much reduced necking than filaments near the wire rim. This indicates that the fracture near the wire axis is of a more brittle nature in these cases. This is the same region where hardness was found to be increased compared with zones near the outer shell. The observation raises the assumption that fracture starts near the softer wire exterior or even in the wire shell, as necking (as an indication of local strain) is further advanced there. After crack propagation becomes critical and the first filaments are torn apart, the load on the remaining filaments increases rapidly, as well as their local strain rate. This might lead to a brittle fracture characteristic of the center filaments and could explain the observations.

It is not quite clear why this behavior is not found in the fracture surface of TiAl3. Two reasons are possible: (i) the crack initiation occurred at a higher load compared with TiAl1 and 2; or (ii) the overall wire configuration is more homogeneous, due to the already small volume fraction of $\mathrm{Al}$ compared to the other wires.

\subsection{Global Yield Stress Calculation}

It has been proven for many kinds of different materials that there is a linear relationship between hardness and yield strength [33,34]. This relationship even holds for cold worked,UFG or NC materials [35]. The linearity only depends on the hardness measurement set up, e.g., the indenter type, indentation force or time.

Since all hardness measurements in this study were carried out under exactly the same conditions, a certain linear correlation should be obtained, as well. In Figure 7, mean values of hardness measurements taken from different composites are shown additionally to the already discussed flow curves and their extrapolations. The turquoise filled diamond symbols correspond to the differently strained Ti parts near the center of the TiAl3 composite. The unfilled symbols represent the equivalent hardness values near the wire rim. Note that they are shifted to lower strain values manually by 0.3 for better visibility, but belong to the same strain level as the filled data points.

It turns out that the observed hardness values of the titanium used near the wire center exactly follow the previously discussed Hockett equation. This gives rise to Hockett's assumption that Ti in this case is strengthened mainly due to cell formation and the approach of a steady-state cell size. Nevertheless, the strong correlation between hardness and the proposed yield stress by Hockett allows the determination of the linearity factor between them. This approach seems justified, since Figure $3 \mathrm{~b}$ showed that $\mathrm{Ti}$ hardness is not influenced by phase boundaries and should therefore be only dependent on the condition of strain hardening. Furthermore, recrystallization or related softening phenomena should be suppressed, due to the low homologous temperature during room temperature deformation. If one now assumes the expression:

$$
H_{N}=k \sigma_{y}
$$

( $H_{N}$, hardness obtained with the described nanoindentation set up; $k$, the linearity factor; $\sigma_{y}$, the true yield stress) to be true, then $k$ is estimated to 4.425 using the four data points shown. The same trend 
is observed for the hardness values in the TiAl2 and TiAl1 composite, but the corresponding data points are not shown in the figure for clarity (they can be found in the Supplementary). Tabor estimates the linearity factor in this equation to a significantly lower value of $k_{T}=2.8$ [36,37]. Despite the fact, that the ISE can increase the $k$-value when dealing with very small indentations, the indentation strain rate $\left(0.05 \mathrm{~s}^{-1}\right)$ was about two orders of magnitude higher than the strain rate during tensile testing $\left(3.3 \times 10^{-4} \mathrm{~s}^{-1}\right)$. Hence, estimated hardness values overestimate the local compared to the macroscopically estimated yield stress. However, the aforementioned problems should be kept in mind, but only affect the absolute value of $k$, not the linear relationship in principle.

Figure 7. A combination of Figure 1 and Figure 3a. Data points with error bars belong to the right axis, providing good agreement for Hockett's equation and TiAl3 titanium hardness.

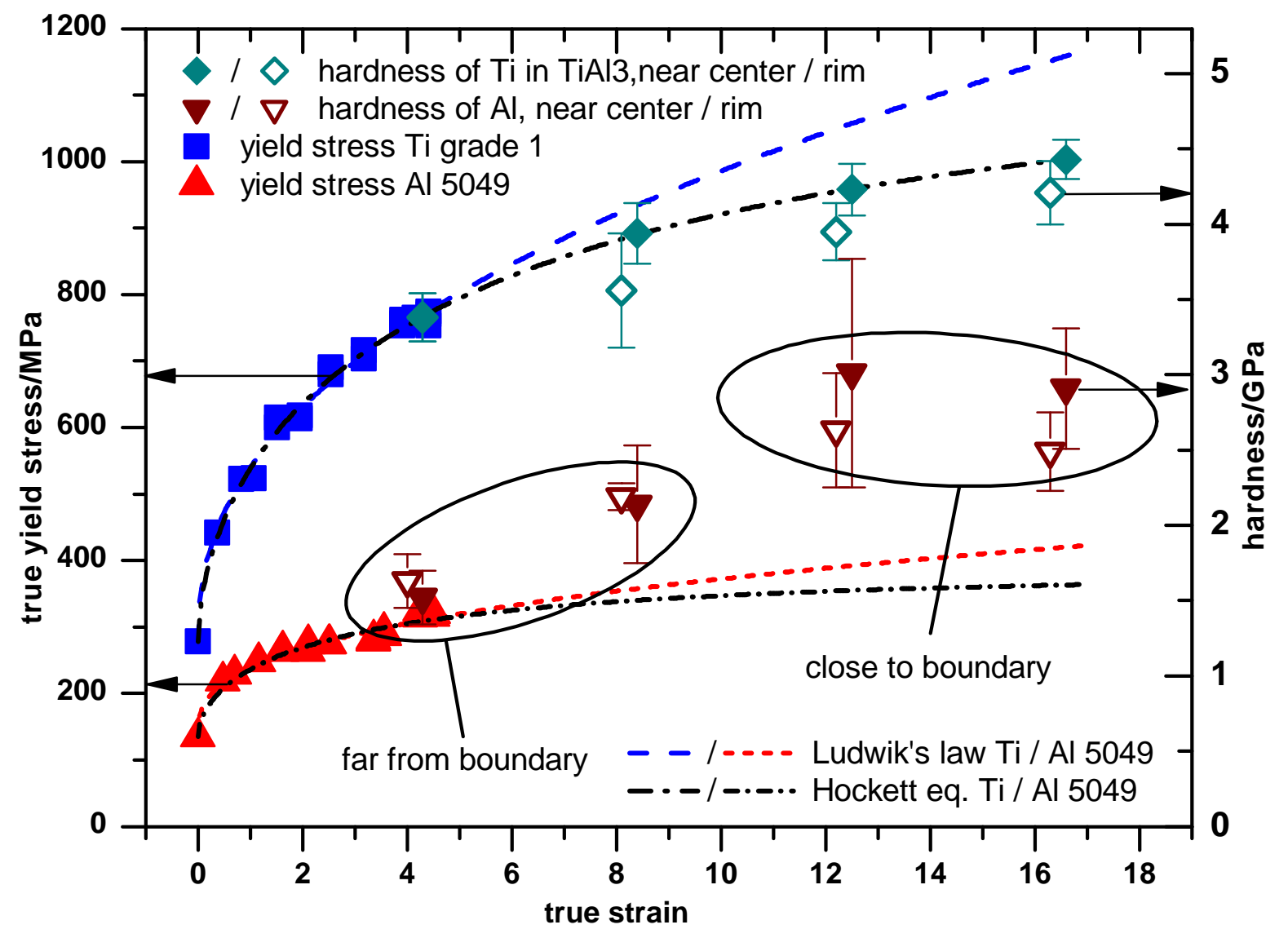

The brown triangle symbols in Figure 7 show the hardness of the Al phase, and for some reason, they do not follow the proposed trends by Ludwik and Hockett and are shifted to higher values then expected. Besides the amount of true strain and the additional Ti shell, the amount of phase boundaries in the composite is the only parameter changed. Therefore, phase boundaries influence the Al hardness level on top of strain.

$\mathrm{Al}$ in TiAl0 ( $\rho=4.3)$ is least affected by phase boundaries, since there is only one in the composite. As a consequence, its hardness (taken far from the boundary) almost meets the measured data by applying Equation (3). In TiAl1 $(\rho=8.4)$, hardness is already shifted to more than $2 \mathrm{GPa}$, even at the highest possible distances to the next phase boundary. This is significantly more than expected, and 
therefore, a noticeable contribution to the hardness on top of strain is active. It was already stated that residual stresses might play a role in this case, since their occurrence could also explain the Ti hardness gradient from the center to the rim. The latter observation is not found for the Al in TiAl0 and TiAll, but for TiAl2 and TiAl3, respectively.

The hardness of $\mathrm{Al}$ in TiAl2 and $\operatorname{TiAl3}(\rho=12.5$ and 16.6) is superimposed by an exaggeration at the phase boundaries, as was shown in Figure 4, and is, therefore, not directly comparable to TiAl0 and TiAl1.

However, if the prior obtained value for $k=4.425$ is taken to be correct in this particular case, the following thought experiment becomes possible: The yield stress of the composite can be thought of as the sum of the yield stresses of all of its parts. Those can be obtained by Equation (3) and the given $k$-value. Then, the corresponding volume fraction of each part is allocated to the obtained yield stress. The total composite yield stress should then be obtained by summing up all parts of the composites.

Since hardness differs from center to the rim of each wire, an arithmetic mean value is calculated from each wire considered completely as the rim and center, respectively. Then, the obtained value is compared to the composite yield stress measured in tension (the diamond symbols in Figure 1). The obtained values are given in Table 3 for comparison.

Table 3. Measured composite yield stresses $\sigma_{y, \text { meas }}$ (mean value of two measurements) compared with the calculated values based on nanoindentation hardness, $\sigma_{y, \text { calc }} . \Delta \sigma_{y, \text { meas }}$ and $\Delta \sigma_{y, c a l c}$ are obtained by a detailed calculation of errors given in the Supplementary.

\begin{tabular}{|c|c|c|c|c|}
\hline & $\sigma_{\mathbf{y}, \text { meas }}$ & $\Delta \sigma_{\mathbf{y}, \text { meas }}$ & $\sigma_{\mathbf{y}, \text { calc }}$ & $\Delta \sigma_{\mathbf{y}, \text { calc }}$ \\
\hline \multicolumn{5}{|c|}{ All units MPa } \\
\hline TiAl0 & 548 & 4 & 537 & 39 \\
\hline TiAl1 & 736 & 5 & $689\left(715^{*}\right)$ & $24\left(25^{*}\right)$ \\
\hline TiAl2 & 781 & 6 & 782 & 29 \\
\hline TiAl3 & 816 & 6 & 811 & 27 \\
\hline
\end{tabular}

The calculated values in the table are in very good agreement with the measured data, especially when taking the additional hardness enhancement in the Al alloy for the TiAl1 composite into account. The corresponding value is given in brackets. A detailed calculation of the errors of the measured and the calculated values is given in the Supplementary, and the results are also given in the table. Although some error bars in Figure 7 would suggest a higher error for the calculated yield stresses, the most likely errors according to Gaussian's law of error propagation are in a reasonable range of $24 \mathrm{MPa}$ to $39 \mathrm{MPa}$.

This good agreement could also be achieved by chance. Nevertheless, the calculation gives very good agreement for all of the differently composed composites, not only TiAl3, from which the $k$-factor is 
derived. From this point of view, the proposed thought experiment gives proof to the assumptions being made, which were: (i) the Hockett equation describes the flow stress of Ti grade 1 up to a true strain of 17; (ii) there is the same linear correlation between hardness and yield stress for both materials; and (iii) the yield stress of the composite can be obtained by adding the yield stresses of the composite parts weighted with their volume fraction.

While (i) and (ii) were already discussed, (iii) seems only possible when yielding starts in every part of the composite at the same time (or the same external load). First of all, this sounds non-credible, since $\mathrm{Ti}$ and $\mathrm{Al}$ are different in their Young's modulus and in their overall flow stress. An external load on a composite of both would certainly lead to one component starting to yield before the other one does.

In fact, this is not the case here when the preparation route of the wires is considered. All parts of the wires are deformed simultaneously during preparation. This means that after a certain threshold strain is reached at the very beginning of the deformation route, all phases yield concurrently. Subsequent to deformation, the wires relax elastically. Due to the different yield stress levels in the different parts and the different elastic modulus, the relaxed state will not cause every wire part to reach a zero stress level, but rather, their sum will do so. Hence, a characteristic residual stress state within each wire is expected. This supports the indications found for residual stress discussed earlier.

Nevertheless, when such a relaxed wire is loaded again, e.g., in a tensile test, all parts of the wires should then reach their yield stress at the same time (external load) again, which makes assumption (iii) plausible. Additionally, when the obtained fracture surfaces in Figure 6 are considered, assumption (iii) is also supported, since almost all filaments show independent necking, which is an indication for simultaneous yielding.

\section{Conclusions}

Using an accumulative swaging and bundling technique, different composite wires made from $\mathrm{Ti}$ and the Al alloy 5049 in a severely plastically deformed condition are prepared. These wires show higher yield stresses than one can expect from the individual flow curves.

For the analysis, flow curves of the individual materials (up to a true strain of 4.5) are inter- and extrapolated using Ludwik's law and Hockett's equation, respectively. Both descriptions show a very good agreement with the experimental data. However, for very high strains up to 17, Hockett's equation fits the hardness data taken from nanoindentation almost perfectly, while Ludwik's law seems to overestimate the true yield strength.

An increase in hardness for the $\mathrm{Al}$ alloy was found, when a phase boundary is nearby. The same effect was not observed for Ti. This hardness increase is thought to arise from: (i) higher dislocation activity, due to localized additional shear deformation; and as a consequence, (ii) smaller grain sizes based on the advanced stage of grain refinement in these areas.

A global hardness gradient was found for almost all wire parts: the center of the wires showed increased hardness, while comparable areas near the rim were softer. It is proposed that a characteristic residual stress state causes this behavior.

Based on local hardness measurements, an attempt was made to calculate the overall yield stress by making three simple assumptions to the yielding behavior of the wires: (i) the Hockett equation describes 
the flow stress of $\mathrm{Ti}$ grade 1 up to a true strain of 17; (ii) there is the same linear correlation between hardness and yield stress for both materials; and (iii) the yield stress of the composite can be obtained by adding the yield stresses of their parts weighted with the volume fraction accordingly.

Despite the fact that the assumptions made are arguable, the global yield stress of all wires could be calculated with reasonable accuracy.

\section{Acknowledgments}

The experimental support of D. Seifert, T. Wolf, M. Frey and H. J. Klauß is gratefully acknowledged. This work has been supported by the Free State of Saxonia in the framework of the European Centre for Emerging Materials and Processes (ECEMP) under contract no. 100111842.

Moreover, V. Maier., H. W. Höppel and M. Göken gratefully acknowledge the funding of the German Research Council (DFG), which, within the framework of its "Excellence Initiative" supports the Cluster of Excellence "Engineering of Advanced Materials" at the University of Erlangen-Nuremberg.

\section{Conflicts of Interest}

The authors declare no conflicts of interest.

\section{References}

1. Valiev, R.Z.; Islamgaliev, R.K.; Alexandrov, I.V. Bulk nanostructured materials from severe plastic deformation. Prog. Mater. Sci. 2000, 45, 103-189.

2. Zhu, Y.; Huang, J.; Gubicza, J.; Ungar, T.; Wang, Y.; Ma, E.; Valiev, R.Z. Nanostructures in Ti processed by severe plastic deformation. J. Mater. Res. 2003, 18, 1908-1917.

3. Langdon, T.G. The principles of grain refinement in equal-channel angular pressing. Mat. Sci. Eng. A 2007, 462, 3-11.

4. Vorhauer, A.; Pippan, R. On the homogeneity of deformation by high pressure torsion. Scr. Mater. 2004, 51, 921-925.

5. Zehetbauer, M.; Kohout, J.; Schafler, E.; Sachslehner, F.; Dubravina, A. Plastic deformation of nickel under high hydrostatic pressure. J. All. Comp. 2004, 378, 329-334.

6. Saito, Y.; Tsuji, N.; Utsunomiya, H.; Sakai, T.; Hong, R.G. Ultra-fine grained bulk aluminum produced by accumulative roll-bonding (ARB) process. Scr. Mater. 1998, 39, 1221-1227.

7. Hausöl, T.; Höppel, H.W.; Göken, M. Microstructure and Mechanical Properties of Accumulative Roll Bonded Aluminium Alloy AA5754. In Journal of Physics Conference Series, Proceeidngs of the ICSMA 15, Dresden, Germany, 16-21 August 2009; Skrotzki, W., Oertel, C., Biermann, H., Heilmaier, M., Eds.; IOP Publishing: Bristol, UK, 2010; Volume 240.

8. Scharnweber, J.; Skrotzki, W.; Oertel, C.G.; Brokmeier, H.G.; Höppel, H.; Topic, I.; Jaschinski, J. Texture, microstructure, mechanical properties of ultrafine-grained Aluminum produced by accumulative roll bonding. Adv. Eng. Mater. 2010, 12, 989-994. 
9. Boehner, A.; Maier, V.; Durst, K.; Höppel, H.W.; Göken, M. Macro- and nanomechanical properties and strain Rate sensitivity of accumulative roll bonded and equal channel angular pressed ultrafine-grained materials. Adv. Eng. Mater. 2011, 13, 251-255.

10. Belyakov, A.; Gao, W.; Miura, H.; Sakai, T. Strain-induced grain evolution in polycrystalline copper during warm deformation. Metall. Mater. Trans. A 1998, 29, 2957-2965.

11. Sitdikov, O.; Sakai, T.; Goloborodko, A.; Miura, H.; Kaibyshev, R. Grain refinement in coarse-grained $7475 \mathrm{Al}$ alloy during severe hot forging. Phil. Mag. 2005, 85, 1159-1175.

12. Beygelzimer, Y.; Orlov, D.; Varyukhin, V. New Severe Plastic Deformation Method: Twist Extrusion. In Ultrafine Grained Materials II, Proceedings of the 2nd International Symposium on Ultrafine Grained Materials, Seattle, WA, USA, 17-21 February 2002; Zhu, Y., Langdon, T., Mishra, R., Semiatin, S., Saran, M., Lowe, T., Minerals, Metals and Materials Society, Mat Process \& Mfg Div, Shaping and Forming Committee; Minerals Met \& Mat, Struct Mat Div, Mechanical Behavior Committee, Eds.; TMS: Seattle, WA, USA, 2002; pp. 297-304. .

13. Bulk Nanostructured Materials; Michael, J., Zehetbauer, Y.T.Z., Eds.; WILEY-VCH: Weinheim, Germany, 2009.

14. Hausöl, T.; Höppel, H.W.; Göken, M. Microstructure and mechanical properties of accumulative roll bonded AA6014/AA5754 aluminium laminates. Mater. Sci. Forum 2011, 667-669, 217-222.

15. Zhang, R.; Acoff, V.L. Processing sheet materials by accumulative roll bonding and reaction annealing from Ti/Al/Nb elemental foils. Mater. Sci. Eng. A 2007, 463, 67-73.

16. Chekhonin, P.; Beausir, B.; Scharnweber, J.; Oertel, C.G.; Hausöl, T.; Höppel, H.W.; Brokmeier, H.G.; Skrotzki, W. Confined recrystallization of high-purity aluminium during accumulative roll bonding of aluminium laminates. Acta Mater. 2012, 60, 4661-4671.

17. Hausöl, T.; Maier, V.; Schmidt, C.; Winkler, M.; Höppel, H.; Göken;, M. Tailoring materials properties by accumulative roll bonding. Adv. Eng. Mater. 2010, 12, 740-746.

18. Marr, T.; Freudenberger, J.; Kauffmann, A.; Scharnweber, J.; Oertel, C.G.; Skrotzki, W.; Siegel, U.; Kuehn, U.; Eckert, J.; Martin, U.; et al. Damascene light-weight metals. Adv. Eng. Mater. 2010, 12, 1191-1197.

19. Marr, T.; Freudenberger, J.; Seifert, D.; Klau $\beta$, H.; Romberg, J.; Okulov, I.; Scharnweber, J.; Eschke, A.; Oertel, C.G.; Skrotzki, W.; et al. Ti-Al composite wires with high specific strength. Metals 2011, 1, 79-97.

20. Oliver, W.; Pharr, G. An improved technique for determining hardness and elastic-modulus using load and displacement sensing indentation experiments. J. Mater. Res. 1992, 7, 1564-1583.

21. Pharr, G.; Oliver, W.; Brotzen, F. On the generality of the relationship among contact stiffness, contact area, and elastic-modulus during indentation. J. Mater. Res. 1992, 7, 613-617.

22. Maier, V.; Höppel, H.W.; Göken, M. Nanomechanical Behavior of Al-Ti Layered Composites Produced by Accumulative Roll Bonding. In Journal of Physics Conference Series, Proceedings of the ICSMA 15, Dresden, Germany, 16-21 August 2009; Skrotzki, W., Oertel, C., Biermann, H., Heilmaier, M., Eds.; IOP Publishing: Bristol, UK, 2010; Volume 240.

23. Hay, J.L.; Pharr, G.M. ASM Handbook: Mechanical Testing; ASM International: Materials Park, OH, USA, 2000; Volume 8. 
24. Ludwik, P. Elemente der Technologischen Mechanik; Springer: Madison, WI, USA, 1909.

25. Hockett, J.; Sherby, O. Large strain deformation of polycrystalline metals at low homologous temperatures. J. Mech. Phys. Sol. 1975, 23, 87-98.

26. Hollomon, J. Tensile deformation. Trans. Am. Inst. Min. Metall. Eng. 1945, 162, 268-290.

27. Marr, T. Hochumgeformte Leichtmetallverbundwerkstoffe und deren festigkeitsbestimmende Faktoren. Ph.D. Thesis, TU Dresden, Dresden, Germany, 2014, submitted.

28. Muller, F. Verfahrensgrundlagen des Rundknetens. Ph.D. Thesis, Berichte aus Produktion und Umformtechnik, Band 36, Technische Hochschule Darmstadt, Shaker Verlag, Aachen, 1997.

29. Nix, W.; Gao, H. Indentation size effects in crystalline materials: A law for strain gradient plasticity. J. Mech. Phys. Sol. 1998, 46, 411-425.

30. Backes, B.; Durst, K.; Goken, M. Determination of plastic properties of polycrystalline metallic materials by nanoindentation: Experiments and finite element simulations. Phil. Mag. 2006, 86, 5541-5551.

31. Durst, K.; Backes, B.; Goken, M. Indentation size effect in metallic materials: Correcting for the size of the plastic zone. Scr. Mater. 2005, 52, 1093-1097.

32. Estrin, Y.; Vinogradov, A. Extreme grain refinement by severe plastic deformation: A wealth of challenging science. Acta Mater. 2013, 61, 782-817.

33. Cahoon, J.; Broughton, W.; Kutzak, A. The determination of yield strength from hardness measurements. Metall. Trans. 1971, 2, 1979-1983.

34. Pavlina, E.; Tyne, C. Correlation of yield strength and tensile strength with hardness for steels. J. Mater. Eng. Perform. 2008, 17, 888-893.

35. Zhang, P.; Li, S.X.; Zhang, Z.F. General relationship between strength and hardness. Mater. Sci. Eng. A 2011, 529, 62-73.

36. Tabor, D. The hardness and strength of metals. J. I. Met. 1951, 79, 1-18.

37. Tabor, D. The Hardness of Metals; Oxford University Press: New York, NY, USA, 1951.

(C) 2014 by the authors; licensee MDPI, Basel, Switzerland. This article is an open access article distributed under the terms and conditions of the Creative Commons Attribution license (http://creativecommons.org/licenses/by/3.0/). 